\title{
Event-triggered sampled-data synchronization of complex networks with time-varying coupling delays
}

\author{
Yang Yang ${ }^{1 *}$ (D) and Yue Long ${ }^{1}$
}

"Correspondence:

yangyang_red@163.com

${ }^{1}$ School of Physics, Liaoning

University, Shenyang, China

\section{Springer}

\begin{abstract}
This paper investigates the event-triggered sampled-data synchronization problem of complex networks with time-varying coupling delays. The sampled-data controller is designed with event-triggered mechanisms. Some results in terms of a linear matrix inequality are obtained to guarantee the asymptotical synchronization of complex networks with time-varying coupling delays. Lastly, we test the effectiveness of the proposed method via some numerical examples.
\end{abstract}

Keywords: Complex networks; Event-triggered; Sampled-data; Synchronization

\section{Introduction}

Recently, many synchronization schemes for complex networks have been proposed. Some synchronization strategies can achieve good performance by various methods [115]. In this paper, the main central point is constricted in the field of sampled-data control due to the development of digital control techniques that has occurred in two decades. Generally, there exist several methods or techniques concerning with digital control. For example, discretization is the most direct approach applied to digital control. However, owing to the complicated dynamics of chaotic Lur'e systems, the exact discrete model is with difficultly obtained. In order to improve the inter-sampling performance, sampleddata control has been often utilized to build hybrid system models via a zero-order hold (ZOH) [7-13]. For example, the sampled-data fuzzy controller was designed for chaotic synchronization in [7] and sampled-data synchronization of chaotic Lur'e systems with time delays was also studied in [13]. However, these schemes have been generally demanded with a fixed sampling period and the actual sampling rate maybe is very high. Aim to solve the above problem, the event-triggered sampled-data controller can be designed to apply for the synchronization of chaotic Lur'e systems. Nowadays, some results for event-triggered control are enumerated, such as [16-18]. Following these novel ideas, the event-triggered communication scheme is considered and the sufficient condition in terms of linear matrix inequality (LMI) is derived to guarantee the asymptotical synchronization of chaotic Lur'e systems. The main contributions of this paper can be described

(c) The Author(s) 2020. This article is licensed under a Creative Commons Attribution 4.0 International License, which permits use sharing, adaptation, distribution and reproduction in any medium or format, as long as you give appropriate credit to the original author(s) and the source, provide a link to the Creative Commons licence, and indicate if changes were made. The images or other third party material in this article are included in the article's Creative Commons licence, unless indicated otherwise in a credit line to the material. If material is not included in the article's Creative Commons licence and your intended use is not permitted by statutory regulation or exceeds the permitted use, you will need to obtain permission directly from the copyright holder. To view a copy of this licence, visit http://creativecommons.org/licenses/by/4.0/. 
as: (1) event-triggered nonuniform sampling is considered in complex networks with timevarying coupling delays; (2) the nonlinear part of the node system is also handled.

\section{Problem formulation}

Consider the following complex networks with time-varying coupling delays consisting of $N$ nodes via an event-triggered control approach:

$$
\begin{aligned}
\dot{x}_{i}(t)= & A x_{i}(t)+B f\left(x_{i}(t)\right)+c \sum_{j=1}^{N} g_{i j} \Gamma x_{j}(t-\tau(t)) \\
& +u_{i}\left(t_{i_{k}}\right), \quad t \in\left[t_{i_{k}}, t_{i_{k+1}}\right),
\end{aligned}
$$

where $i=1,2, \ldots, N, N$ is the number of nodes, $k=0,1, \ldots, \infty, x_{i}(t) \in \mathbb{R}^{n}$ denotes the state vector associated with the $i$ th node, $t_{i_{k}}$ denotes the event-triggered instant and it is determined by the transmission error and the state error, $u_{i}\left(t_{i_{k}}\right) \in \mathbb{R}^{n}$ denotes the designed control law where the transmitted data packets are utilized along with the event-triggered control happening, $\tau(t)$ denotes the time-varying delay and satisfies the condition $\dot{\tau}(t)<v \leq 1$, $A \in \mathbb{R}^{n \times n}, B \in \mathbb{R}^{n \times m}$ are known constant matrices, $f: \mathbb{R}^{n} \rightarrow \mathbb{R}^{m}$ is a continuous vectorvalued function, $c$ is a constant coupling strength, $\Gamma \in \mathbb{R}^{n \times n}$ denotes the inner coupling matrix, $G=\left(g_{i j}\right)_{N \times N}$ is the coupling configuration matrix: if nodes $i$ and $j(i \neq j)$ are connected, then $g_{i j}>0$, otherwise $g_{i j}=0$, the diagonal elements of matrix $G$ are defined by $g_{i i}=-\sum_{j=1, i \neq j}^{N} g_{i j}, i=1,2, \ldots, N . t_{k}$ denotes the whole sampling instant and it is irregular. Moreover, the serial number of transmitted data packets, denoted as $i_{k}$, may be discontinuous for the existence of event-triggered mechanism where the transmitted data packets refer to the ones successfully arriving at the plant. $i_{k} \in \mathbb{N}$ denotes the serial number of the transmitted data packet such that $\left\{i_{0}, i_{1}, i_{2}, \ldots\right\} \subseteq\{0,1,2,3, \ldots\}$.

Let $e_{i}(t)=x_{i}(t)-s(t)$ be the error vectors, where $s(t) \in \mathbb{R}^{n}$ is a solution of a target node satisfying $\dot{s}(t)=A s(t)+B f(s(t))$. The sampled-data synchronization feedback controller is designed as

$$
u_{i}\left(t_{i_{k}}\right)=K_{i} e_{i}\left(t_{i_{k}}\right), \quad t \in\left[t_{i_{k}}, t_{i_{k+1}}\right),
$$

where $K_{i}$ is the feedback gain matrix with appropriate dimensions. Then, the error dynamics of (1) can be obtained as follows:

$$
\begin{aligned}
\dot{e}_{i}(t)= & A e_{i}(t)+B g\left(e_{i}(t)\right)+c \sum_{j=1}^{N} g_{i j} \Gamma e_{j}(t-\tau(t)) \\
& +K_{i} e_{i}\left(t_{i_{k}}\right), \quad t \in\left[t_{i_{k}}, t_{i_{k+1}}\right),
\end{aligned}
$$

where $i=1,2, \ldots, N$ and $g\left(e_{i}(t)\right)=f\left(x_{i}(t)\right)-f(s(t))$. It is clear that (3) can be rewritten in a vector-matrix form,

$$
\begin{aligned}
\dot{e}(t)= & A_{N} e(t)+B_{N} \bar{g}(e(t))+c(G \otimes \Gamma) e(t-\tau(t)) \\
& +K e\left(t_{i_{k}}\right), \quad t \in\left[t_{i_{k}}, t_{i_{k+1}}\right),
\end{aligned}
$$

where $e(t)=\left[e_{1}^{T}(t), \ldots, e_{N}^{T}(t)\right]^{T}, \bar{g}(e(t))=\left[g^{T}\left(e_{1}(t)\right), \ldots, g^{T}\left(e_{N}(t)\right)\right]^{T}, K=\operatorname{diag}\left\{K_{1}, \ldots, K_{N}\right\}$, $A_{N}=I_{N} \otimes A, B_{N}=I_{N} \otimes B$. 
The error between the current sampling instant and the latest transmission instant can be calculated as $\bar{e}\left(t_{i_{k}+l}\right)=e\left(t_{i_{k}+l}\right)-e\left(t_{i_{k}}\right)$ where $l=1,2, \ldots, d, d=i_{k+1}-i_{k}$. The eventtriggered condition can be provided as

$$
\bar{e}^{T}(t) \Phi \bar{e}(t) \geq \delta e^{T}(t) \Phi e(t)
$$

where $\delta>0$ is a given scalar parameter, $\Phi$ is a positive-definite weighting matrix with appropriate dimensions. If the event-triggered condition is satisfied, then the transmitted signals will be sent. Otherwise the sampling signals will not be sent. $\Omega:=\left[t_{i_{k}}, t_{i_{k}+l}\right)$ can be described as $\Omega=\bigcup_{l=1}^{d} \Omega_{l}$ where $\Omega_{l}:=\left[t_{i_{k}+l-1}, t_{i_{k}+l}\right)$.

Assumption 1 There exists a constant $h>0$ such that

$$
t_{k+1}-t_{k} \leq h, \quad k=0,1, \ldots, \infty
$$

where $h$ denotes the upper bound of the interval between two consecutive sampling instants.

Assumption 2 ([19]) There exists a diagonal matrix $\Lambda=\operatorname{diag}\left\{\lambda_{1}, \ldots, \lambda_{N}\right\}>0$ such that the nonlinear part of the node system satisfies the following condition in the domain of definition:

$$
(f(x)-f(y))^{T}(f(x)-f(y)) \leq(x-y)^{T} \Lambda(x-y) .
$$

Combining (4) and (5), the final error dynamics can be described as

$$
\begin{aligned}
\dot{e}(t)= & A_{N} e(t)+B_{N} \bar{g}(e(t))+c(G \otimes \Gamma) e(t-\tau(t)) \\
& +K\left(e(t-d(t))-\bar{e}\left(t_{i_{k}+l}\right)\right), \quad t \in \Omega_{l},
\end{aligned}
$$

where $d(t)=t-t_{i_{k}+l}$. It is explicit that $-h \leq d(t)<0$ due to $\Omega_{l}$ and (6). The control objective is to design the controller gain matrix $K$ such that the error dynamics (8) is asymptotically stable, i.e., $e(t) \rightarrow 0$ as $t \rightarrow \infty$.

\section{Main results}

In this section, the event-triggered sampled-data synchronization scheme will be given.

Theorem 1 Given the scalars $h>0, \delta>0$, the error dynamics (8) is global asymptotically stable concerning with the event-triggered condition (5), if there exist matrices $P=P^{T}>0$, $Q=Q^{T}>0, R=R^{T}>0, \Phi=\Phi^{T}>0, \Lambda=\operatorname{diag}\left\{\lambda_{1}, \ldots, \lambda_{N}\right\}>0$, any matrices $N_{1}, N_{2}, N_{3}$, $M_{1}, M_{2}, M_{3}$ with appropriate dimensions, and the controller gain matrix $K$, such that the following condition holds:

$$
\left[\begin{array}{cc}
\Theta & h N \\
* & -h R
\end{array}\right]<0,
$$


where

$$
\begin{aligned}
& \Theta=\left[\begin{array}{ccccc}
\Phi_{11} & \Phi_{12} & \Phi_{13} & \Phi_{14} & \Phi_{15} \\
* & \Phi_{22} & \Phi_{23} & \Phi_{24} & \Phi_{25} \\
* & * & \Phi_{33} & \Phi_{34} & \Phi_{35} \\
* & * & * & \Phi_{44} & 0 \\
* & * & * & * & \Phi_{55}
\end{array}\right], \quad N=\left[\begin{array}{c}
N_{1} \\
N_{2} \\
N_{3} \\
0 \\
0
\end{array}\right], \\
& \Phi_{11}=Q+N_{1}+N_{1}^{T}+M_{1} A_{N}+A_{N}^{T} M_{1}^{T}+M_{1} B_{N} B_{N}^{T} M_{1}^{T}+3 \Lambda, \\
& \Phi_{12}=P+N_{2}^{T}-M_{1}+A_{N}^{T} M_{2}^{T}, \\
& \Phi_{13}=-N_{1}+N_{3}^{T}+M_{1} K+A_{N}^{T} M_{3}^{T}, \\
& \Phi_{14}=c M_{1}(G \otimes \Gamma), \\
& \Phi_{15}=-M_{1} K, \\
& \Phi_{22}=h R-M_{2}-M_{2}^{T}+M_{2} B_{N} B_{N}^{T} M_{2}^{T}, \\
& \Phi_{23}=-N_{2}+M_{2} K-M_{3}^{T}, \\
& \Phi_{24}=c M_{2}(G \otimes \Gamma), \\
& \Phi_{25}=-M_{2} K \\
& \Phi_{33}=-N_{3}-N_{3}^{T}+M_{3} K+K^{T} M_{3}^{T}+M_{3} B_{N} B_{N}^{T} M_{3}^{T}+\delta \Phi, \\
& \Phi_{34}=c M_{3}(G \otimes \Gamma), \\
& \Phi_{3}=-(1-v) Q, \\
& \Phi_{1},
\end{aligned}
$$

Proof Construct the following Lyapunov-Krasovskii functional:

$$
V(t)=e^{T}(t) \operatorname{Pe}(t)+\int_{t-\tau(t)}^{t} e^{T}(s) Q e(s) d s+\int_{-h}^{0} \int_{t-\theta}^{t} \dot{e}^{T}(s) \operatorname{Re}(s) d s d \theta
$$

where $P=P^{T}>0, Q=Q^{T}>0$, and $R=R^{T}>0$. Moreover, the following equations hold for any appropriate dimensional matrices $N_{j}(j=1,2, \ldots, 3)$ and $M_{l}(l=1,2,3)$ :

$$
\begin{aligned}
& {\left[e^{T}(t) N_{1}+\dot{e}^{T}(t) N_{2}+e^{T}(t-d(t)) N_{3}\right]} \\
& \quad \times\left[e(t)-e(t-d(t))-\int_{t-d(t)}^{t} \dot{e}(s) d s\right]=0, \\
& {\left[e^{T}(t) M_{1}+\dot{e}^{T}(t) M_{2}+e^{T}(t-d(t)) M_{3}\right] \times\left[-\dot{e}(t)+A_{N} e(t)+B_{N} \bar{g}(e(t))\right.} \\
& \left.\quad+c(G \otimes \Gamma) e(t-\tau(t))+K e(t-d(t))-K \bar{e}\left(t_{i_{k}+l}\right)\right]=0 .
\end{aligned}
$$


Combining (10) and (11), the corresponding time derivative of $V(t)$ is given by

$$
\begin{aligned}
\dot{V}(t) \leq & 2 \dot{e}^{T}(t) P e(t)+e^{T}(t) Q e(t)-(1-v) e^{T}(t-\tau(t)) Q e(t-\tau(t)) \\
& +h \dot{e}^{T}(t) \operatorname{Re}(t)+\int_{t+h}^{t} \dot{e}^{T}(s) \operatorname{Re}(s) d s+2\left[e^{T}(t) N_{1}+\dot{e}^{T}(t) N_{2}\right. \\
& \left.+e^{T}(t-d(t)) N_{3}\right] \times\left[e(t)-e(t-d(t))-\int_{t-d(t)}^{t} \dot{e}(s) d s\right] \\
& +2\left[e^{T}(t) M_{1}+\dot{e}^{T}(t) M_{2}+e^{T}(t-d(t)) M_{3}\right] \times\left[-\dot{e}(t)+A_{N} e(t)\right. \\
& \left.+B_{N} \bar{g}(e(t))+c(G \otimes \Gamma) e(t-\tau(t))+K e(t-d(t))-K \bar{e}\left(t_{i_{k}+l}\right)\right] \\
& +\bar{e}^{T}\left(t_{i_{k}+l}\right) \Phi \bar{e}\left(t_{i_{k}+l}\right)-\bar{e}^{T}\left(t_{i_{k}+l}\right) \Phi \bar{e}\left(t_{i_{k}+l}\right) .
\end{aligned}
$$

Utilizing Lemma 1 and Lemma 2 in [20], (5), and (7), the following result can be obtained:

$$
\dot{V}(t) \leq \varsigma^{T}(t)\left(\Theta+h N R^{-1} N^{T}\right) \varsigma(t)
$$

where $\zeta(t)=\left[e^{T}(t) \dot{e}^{T}(t) e^{T}(t-d(t)) e^{T}(t-\tau(t)) \bar{e}^{T}\left(t_{i_{k}+l}\right)\right]^{T}$, matrices $\Theta$ and $N$ are defined in (9). It is explicit that, if $\Theta+h N R^{-1} N^{T}<0$ holds, then $\dot{V}(t)<0$ for any nonzero $\varsigma(t)$. Using the Schur complement [21], the result in Theorem 1 can be obtained, and the proof is completed.

Note that the condition (9) in Theorem 1 is not a LMI. Thus, set $M_{1}=J, M_{2}=\epsilon_{1} J, M_{3}=$ $\epsilon_{2} J$ and $J K=V$. Utilizing the Schur complement, the following corollary is obtained.

Corollary 1 Given the scalars $\epsilon_{1}>0, \epsilon_{2}>0, h>0, \delta>0$, the error dynamics (8) is global asymptotically stable concerning with the event-triggered condition (5), if there exist matrices $P=P^{T}>0, Q=Q^{T}>0, R=R^{T}>0, \Phi=\Phi^{T}>0, \Lambda=\operatorname{diag}\left\{\lambda_{1}, \ldots, \lambda_{N}\right\}>0$, any matrices $N_{1}, N_{2}, N_{3}$, J, and $V$ with appropriate dimensions, such that the following condition holds:

$$
\left[\begin{array}{cc}
\bar{\Theta} & h \bar{N} \\
* & -h R
\end{array}\right]<0,
$$

where

$$
\begin{aligned}
\bar{\Theta}=\left[\begin{array}{cccccccc}
\bar{\Phi}_{11} & \bar{\Phi}_{12} & \bar{\Phi}_{13} & \bar{\Phi}_{14} & \bar{\Phi}_{15} & \bar{\Phi}_{16} & 0 & 0 \\
* & \bar{\Phi}_{22} & \bar{\Phi}_{23} & \bar{\Phi}_{24} & \bar{\Phi}_{25} & 0 & \bar{\Phi}_{27} & 0 \\
* & * & \bar{\Phi}_{33} & \bar{\Phi}_{34} & \bar{\Phi}_{35} & 0 & 0 & \bar{\Phi}_{38} \\
* & * & * & \bar{\Phi}_{44} & 0 & 0 & 0 & 0 \\
* & * & * & * & \bar{\Phi}_{55} & 0 & 0 & 0 \\
* & * & * & * & * & \bar{\Phi}_{66} & 0 & 0 \\
* & * & * & * & * & * & \bar{\Phi}_{77} & 0 \\
* & * & * & * & * & * & * & \bar{\Phi}_{88}
\end{array}\right], \quad \bar{N}=\left[\begin{array}{c}
N_{1} \\
N_{2} \\
N_{3} \\
0 \\
0 \\
0 \\
0 \\
0
\end{array}\right], \\
\bar{\Phi}_{11}=Q+N_{1}+N_{1}^{T}+J A_{N}+A_{N}^{T} J^{T}+3 \Lambda, \\
\bar{\Phi}_{12}=P+N_{2}^{T}-J+\epsilon_{1} A_{N}^{T} J^{T}, \\
\bar{\Phi}_{13}=-N_{1}+N_{3}^{T}+V+\epsilon_{2} A_{N}^{T} J^{T}
\end{aligned}
$$




$$
\begin{aligned}
& \bar{\Phi}_{14}=c J(G \otimes \Gamma), \\
& \bar{\Phi}_{15}=-V, \\
& \bar{\Phi}_{16}=J B_{N}, \\
& \bar{\Phi}_{22}=h R-J-J^{T}, \\
& \bar{\Phi}_{23}=-N_{2}+\epsilon_{1} V-\epsilon_{2} J^{T}, \\
& \bar{\Phi}_{24}=c \epsilon_{1} J(G \otimes \Gamma), \\
& \bar{\Phi}_{25}=-\epsilon_{1} V, \\
& \bar{\Phi}_{27}=\epsilon_{1} J B_{N}, \\
& \bar{\Phi}_{33}=-N_{3}-N_{3}^{T}+\epsilon_{2} V+\epsilon_{2} V^{T}+\delta \Phi, \\
& \bar{\Phi}_{34}=c \epsilon_{2} J(G \otimes \Gamma), \\
& \bar{\Phi}_{35}=-\epsilon_{2} V, \\
& \bar{\Phi}_{38}=\epsilon_{2} J B_{N}, \\
& \bar{\Phi}_{44}=-(1-v) Q, \\
& \bar{\Phi}_{55}=-\Phi, \\
& \bar{\Phi}_{66}=-I, \\
& \bar{\Phi}_{77}=-I, \\
& \bar{\Phi}_{88}=-I .
\end{aligned}
$$

Moreover, the sampled-data controller gain is given by $K=J^{-1} V$.

\section{Numerical examples}

In this section, two numerical examples are given to demonstrate the effectiveness of the proposed event-triggered sampled-data synchronization scheme.

Example 1 ([22]) A complex network with five coupled identical nodes is described as follows:

$$
\dot{x}_{i}(t)=A x_{i}(t)+B f\left(x_{i}(t)\right)+\sum_{j=1}^{5} g_{i j} x_{j}(t-\tau(t))+u_{i}(t), \quad i=1,2, \ldots, 5,
$$

where

$$
\begin{aligned}
A & =\left[\begin{array}{ccc}
-a m_{1} & a & 0 \\
1 & -1 & 1 \\
0 & -b & 0
\end{array}\right], \quad B=\left[\begin{array}{ccc}
-a\left(m_{0}-m_{1}\right) & 0 & 0 \\
0 & 0 & 0 \\
0 & 0 & 0
\end{array}\right], \\
G & =0.2 \times\left[\begin{array}{ccccc}
-3 & 1 & 1 & 0 & 1 \\
1 & -4 & 1 & 1 & 1 \\
1 & 1 & -3 & 1 & 0 \\
0 & 1 & 1 & -3 & 1 \\
1 & 1 & 0 & 1 & -3
\end{array}\right],
\end{aligned}
$$




$$
\begin{aligned}
& f(t)=0.5\left(\left|x_{i k}(t)+c\right|-\left|x_{i k}(t)-c\right|\right), \quad k=1, \ldots, 3, \\
& a=9, \quad b=14.28, \quad c=1, \quad m_{0}=-1 / 7, \quad m_{1}=2 / 7, \\
& \tau(t)=0.4+0.1 \sin (t), \quad v=0.1 .
\end{aligned}
$$

Setting $\epsilon_{1}=\epsilon_{2}=1$, and $\delta=0.1$, the condition (12) in Corollary 1 is feasible and the following result is obtained:

$$
\begin{aligned}
& h_{\max }=0.18, \\
& K=\operatorname{diag}\left\{K_{1}, K_{2}, K_{3}, K_{4}, K_{5}\right\},
\end{aligned}
$$

where

$$
\begin{aligned}
& K_{1}=K_{3}=K_{4}=K_{5}=\left[\begin{array}{ccc}
-2.0710 & 4.5512 & 0.3642 \\
0.4899 & -1.8005 & 0.6312 \\
-0.3439 & -9.3591 & -1.4639
\end{array}\right], \\
& K_{2}=\left[\begin{array}{ccc}
-2.1161 & 4.6243 & 0.3494 \\
0.5075 & -1.8259 & 0.6373 \\
-0.3655 & -9.3363 & -1.4738
\end{array}\right] .
\end{aligned}
$$

Example 2 ([22]) Complex network with three coupled identical nodes is described as follows:

$$
\dot{x}_{i}(t)=A x_{i}(t)+B f\left(x_{i}(t)\right)+\sum_{j=1}^{3} g_{i j} x_{j}(t-\tau(t))+u_{i}(t), \quad i=1,2, \ldots, 3,
$$

where

$$
\begin{aligned}
& A=\left[\begin{array}{cc}
-0.5 & 0.2 \\
0 & 0.95
\end{array}\right], \quad B=\left[\begin{array}{cc}
1 & 0 \\
0 & -1
\end{array}\right], \quad G=0.5 \times\left[\begin{array}{ccc}
-1 & 0 & 1 \\
0 & -1 & 1 \\
1 & 1 & -2
\end{array}\right], \\
& f(t)=\left[\begin{array}{c}
\tanh \left(0.2 x_{i 1}(t)\right) \\
\tanh \left(0.75 x_{i 2}(t)\right)
\end{array}\right], \quad \tau(t)=0.2+0.05 \sin (10 t), \quad v=0.05 .
\end{aligned}
$$

Setting $\epsilon_{1}=\epsilon_{2}=1$, and $\delta=0.1$, the condition (12) in Corollary 1 is feasible and the following result is obtained:

$$
\begin{aligned}
& h_{\max }=0.18, \\
& K=\operatorname{diag}\left\{K_{1}, K_{2}, K_{3}\right\},
\end{aligned}
$$

where

$$
K_{1}=K_{2}=\left[\begin{array}{cc}
-2.7786 & 0.0222 \\
-0.1421 & -2.9535
\end{array}\right], \quad K_{3}=\left[\begin{array}{cc}
-2.8814 & 0.0291 \\
-0.1212 & -2.9829
\end{array}\right] \text {. }
$$


Through the above two numerical examples, we can find that the event-triggered sampled-data synchronization is guaranteed for complex networks with time-varying coupling delays.

\title{
5 Conclusion
}

In the present work, we deal with the event-triggered sampled-data synchronization problem of complex networks with time-varying coupling delays. In future work, more performance requirements for the event-triggered sampled-data synchronization of complex networks will be considered in a uniform network topological structure.

\author{
Acknowledgements \\ Not applicable. \\ Funding \\ This work is supported by the Natural Science Foundation of Liaoning Province under Grant 2019-MS-150.
}

Availability of data and materials

Please contact the authors for data requests.

\section{Competing interests}

The authors declare that they have no competing interests.

\section{Authors' contributions}

The simulation experiments are carried out by $Y Y$ and $L Y$, and $Y Y$ was a major contributor in writing the manuscript. All authors read and approved the final manuscript.

\section{Publisher's Note}

Springer Nature remains neutral with regard to jurisdictional claims in published maps and institutional affiliations.

Received: 13 April 2020 Accepted: 2 June 2020 Published online: 24 June 2020

\section{References}

1. Lu, J.G., Hill, D.J.: Impulsive synchronization of chaotic Lur'e systems by linear static measurement feedback: an Imi approach. IEEE Trans. Circuits Syst. II, Express Briefs 54, 710-714 (2007)

2. Chen, W.H., Lu, X., Chen, F.: Impulsive synchronization of chaotic Lur'e systems via partial states. Phys. Lett. A 372, 4210-4216 (2008)

3. Han, Q.L.: On designing time-varying delay feedback controllers for master-slave synchronization of Lur'e systems. IEEE Trans. Circuits Syst. I 54, 1573-1583 (2007)

4. Li, T., Yu, J., Wang, Z.: Delay-range-dependent synchronization criterion for Lur'e systems with delay feedback control. Commun. Nonlinear Sci. Numer. Simul. 14, 1796-1803 (2009)

5. Guo, H., Zhong, S., Gao, F.: Design of pd controller for master-slave synchronization of Lur'e systems with time-delay. Appl. Math. Comput. 212, 86-93 (2009)

6. Yin, C., Zhong, S.M., Chen, W.F.: Design pd controller for master-slave synchronization of chaotic Lur'e systems with sector and slope restricted nonlinearities. Commun. Nonlinear Sci. Numer. Simul. 16, 1632-1639 (2011)

7. Lam, H.K., Seneviratne, L.D.: Chaotic synchronization using sampled-data fuzzy controller based on fuzzy-model-based approach. IEEE Trans. Circuits Syst. I, Regul. Pap. 55, 883-892 (2008)

8. Lu, J.G., Hill, D.J.: Global asymptotical synchronization of chaotic Lur'e systems using sampled data: a linear matrix inequality approach. IEEE Trans. Circuits Syst. II, Express Briefs 55, 586-590 (2008)

9. Zhang, C.K., He, Y., Wu, M.: Improved global asymptotical synchronization of chaotic Lur'e systems with sampled-data control. IEEE Trans. Circuits Syst. II, Express Briefs 56, 320-324 (2009)

10. Chen, W., Wang, Z., Lu, X.: On sampled-data control for master-slave synchronization of chaotic Lur'e systems. IEEE Trans. Circuits Syst. II, Express Briefs 59, 515-519 (2012)

11. Theesar, S., Jeeva, S., Banerjee, S., Balasubramaniam, P.: Synchronization of chaotic systems under sampled-data control. Nonlinear Dyn. 70, 1977-1987 (2012)

12. Zhang, C.K., Jiang, L., He, Y., Wu, Q.H., Wu, M.: Asymptotical synchronization for chaotic Lur'e systems using sampled-data control. Commun. Nonlinear Sci. Numer. Simul. 18, 2743-2751 (2013)

13. Wu, Z.G., Shi, P., Su, H.Y., Chu, J.: Sampled-data synchronization of chaotic Lur'e systems with time delays. IEEE Trans. Neural Netw. Learn. Syst. 24, 410-421 (2013)

14. Chen, X.Y., Huang, T.W., Cao, J.D.: Finite-time multi-switching sliding mode synchronization for multiple uncertain complex chaotic systems with network transmission mode. IET Control Theory Appl. 13(9), 1246-1257 (2019)

15. Chen, X.Y., Park, J.H., Cao, J.D.: Sliding mode synchronization of multiple chaotic systems with uncertainties and disturbances. Appl. Math. Comput. 308, 161-173 (2017)

16. Tabuada, P.: Event-triggered real-time scheduling of stabilizing control tasks. IEEE Trans. Autom. Control $\mathbf{5 2 ,}$ 1680-1685 (2007) 
17. Wang, X., Lemmon, M.D.: Event-triggering in distributed networked control systems. IEEE Trans. Autom. Control 56, 586-601 (2011)

18. Peng, C., Yang, T.C.: Event-triggered communication and $h_{\infty}$ control co-design for networked control systems. Automatica 49, 1326-1332 (2013)

19. Wang, Y.W., Xiao, J.W., Wen, C., Guan, Z.H.: Synchronization of continuous dynamical networks with discrete-time communications. IEEE Trans. Neural Netw. 22, 1979-1986 (2011)

20. Yang, D.D.: Self-synchronization of coupled chaotic Fitzhugh-Nagumo systems with unreliable communication links. Commun. Nonlinear Sci. Numer. Simul. 18, 2783-2789 (2013)

21. Boyd, S., Ghauoi, L.E., Feron, E., Balakrishan, V. (eds.): Linear Matrix Inequalities in System and Control Theory. SIAM, Philadelphia (1994)

22. Lee, T.H., Wu, Z., Park, J.: Synchronization of a complex dynamical network with coupling time-varying delays via sampled-data control. Appl. Math. Comput. 219, 1354-1366 (2012)

Submit your manuscript to a SpringerOpen ${ }^{0}$ journal and benefit from:

- Convenient online submission

- Rigorous peer review

Open access: articles freely available online

- High visibility within the field

- Retaining the copyright to your article

Submit your next manuscript at $\boldsymbol{\nabla}$ springeropen.com 